\title{
Virtual power plant: state of the art providing energy flexibility to local distribution grids
}

\author{
Zahid Ullah $^{1 *}$, and Nayyar Hussain Mirjat ${ }^{2}$ \\ ${ }^{1}$ Institute for Globally Distributed Open Research and Education (IGDORE), Cleveland, Middlesbrough, UK \\ ${ }^{2}$ Mehran University of Engineering \& Technology, Jamshoro, Sindh, PAKISTAN
}

\begin{abstract}
Integrating decentralised energy sources into the traditional distribution networks can result in technical issues impacting the power quality. Innovative ideas are, therefore, needed to promote the transformation of systems to a smart grid. Distribution System operator (DSO) could make use of the flexibility of emerging technologies as a method to address these power quality issues. This study aims to present an overview of a local flexibility market (LFM) which will allow DSO requirements to be fulfilled through the (VPP) as an energy flexibility provider. The required optimization loads, generators and as well as storage units, are undertaken in the general algebraic modeling simulation (GAMS) environment. The aim of the optimization problem is to provide DSOs the opportunity to increase or curtail the local generations and loads in order to satisfy their requirement. The VPP will then be responsible for handling the relevant requests in real time to ensure the correct operating schedule of a resource is applied. The preliminary results of simulation studies presented in this paper have shown that the local market framework for flexibility could have potential for deferring investments in distribution network capacity, minimizing energy costs and improving the hosting capacity of distribution networks.
\end{abstract}

\section{Introduction}

\subsection{Background and aim}

Continuous emission reductions in the European energy system through the adoption of decentralised renewable energy sources has generated worldwide interest in local energy markets for local energy societies [1,]. It is planned that millions of energy and flexibility services providers, hybrid vehicles and storage facilities would be able to provide energy and flexibility services in the European electricity market by 2030 [2]. Their main focus and involvement will be on distribution grids. The local energy community (LEC) definition for the internal electricity market has recently been integrated into Article 16 of the European regulatory authorities and now thought to be an efficient means of regulating energy at community level [3]. As a result, global interest in LEC administration by LFM is increasing and also attracted the attention of policymakers, regulators and scientists alike. LECs have the potential to engage end-users in emerging renewable energy opportunities in the future. To sum up, the idea is to present an overview of a centralised energy market, geographically spread to hundreds of local markets, benefiting local communities and prosumers.

\subsection{A brief literature review and our contributions}

In the recent contemporary literature, several researchers have investigated the issue of operations of LFM. The majority of researchers have suggested an optimization problem formulation towards cost minimization of decentralized energy sources. The authors have suggested an optimization problem formulation in [4,5], minimizing the energy costs in scheduling decentralised energy sources for the benefits of local energy community. In [6], the authors have conducted a case study on the use of flexibility to minimise energy bills from the viewpoint of the prosumer. In [7,8], the authors have proposed an efficient grid-congestion management power flow algorithm with flexible assets. The authors have evaluated numerous local trading concepts in $[9,10]$, enabling the techniques and the necessary mechanism. In [11], the authors have assessed a distributed control strategy focused on the market that divides the grid into nodes and uses smart automatic devices to maximize household benefits. In this case, the system operator is responsible for conducting energy auctions in order to maximize the total surplus of the market without considering LFM services. In [12], the authors have proposed an auction-based LFM model without analysing local market flexibility services. A bidding based LFM, focused on the day Ahead market and Intraday market, is proposed in [13], however, the local market operator interactions with the TSO have not been considered. With respect to the reviewed literature, the contributions of this paper are: proposal of local flexibility market structure presented here which is viewed as a solution that encourage the direct involvement of end-users in the acquisition and sale of energy flexibility through local incentive measures for LEC/prosumers. This LFM framework is thought of to be capable of satisfying DSO demands through the use

*Corresponding author: Zahid.ullah@igdore.org 
of a VPP interface, that offers both collective and individual flexibility services to local community.

\subsection{Paper Layout}

The rest of the paper is structured as follows: Section 2 presents VPP decision making model description, its assumptions and as will as problem formulation. Case study simulation results are discussed in section 3 . Finally, meaningful conclusions are made in section 4.

\section{VPP decision making model}

\subsection{Model description}

The LFM agents and their interaction with each other is shown through Fig. 1.

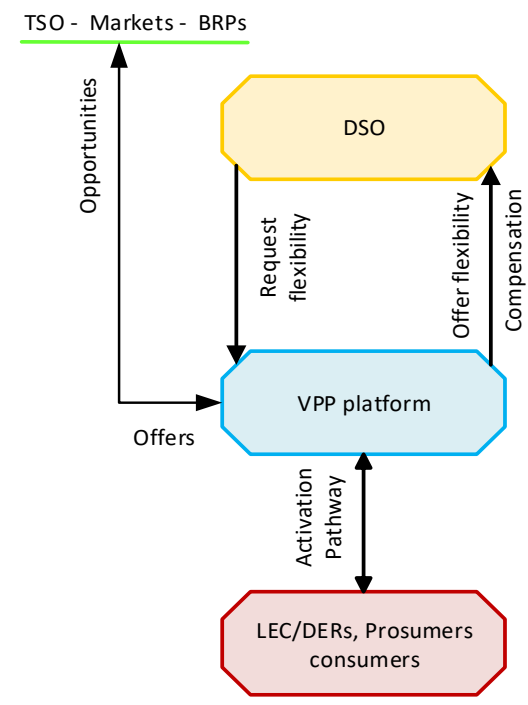

Fig. 1. Local flexibility market agents

- The DSO, buys flexibility services from a VPP platform and in return provides economic benefits.

- The VPP is a flexibility services provider platform, receives offers and requests for flexibility.

- Local energy community is a source of flexibility, including distributed energy resources (DERs), energy cooperatives and prosumers who submit their offers of flexibility.

DERs, prosumers and energy cooperatives are bringing up their flexibility strengths to the VPP network, which competes for the resulting DSO profit

Fig. 2, clearly shows market flexibility contract deal of a customer based on four smart flexible appliances, arranged between the least to the costlier offer, including a dish-washer, washer-dryer, space heater and electric water heater.

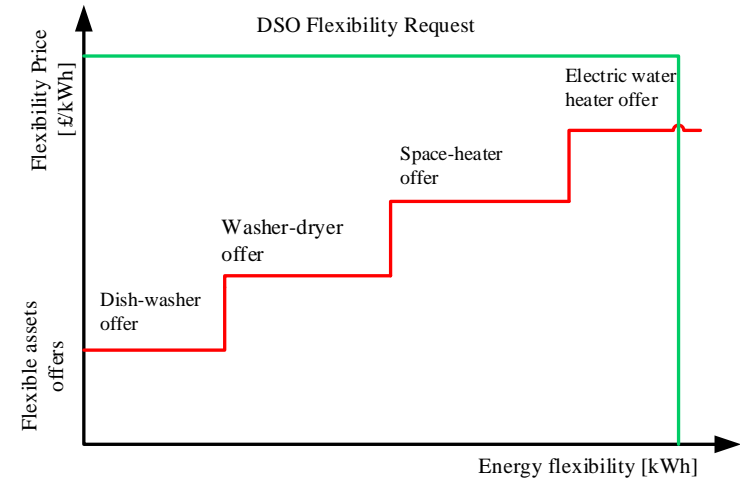

Fig. 2. flexibility example of a customer

\subsection{Model Assumptions}

- It is supposed that VPP is centrally controlled as a smart energy flexibility services provider for the benefits of DSO, local energy communities and prosumers.

- The main participants of local flexibility market are a VPP, the DSO, decentralized generators, prosumers and storage units.

- Local market participants need to have necessary standard contract with the VPP and hence direct trade between traders is not allowed.

- All participants of LFM submit their offer of energy flexibility services to the VPP in the form of blocks.

- The VPP trades energy flexibility services as an intermediary to the local DSO on behalf of LECs/prosumers and get financial benefits in return based on flexibility agreement and the VPP decision.

A smart communication metering device (SCMD) is required for all flexible participants with DER resources. The SCMD records each flexible participant energy generation and consumption. This SCMD must be able to receive the command signal from the VPP data analysis centre.

\subsection{Objective Function}

The overall operational cost function minimisation model of VPP in a short-term planning horizon is formulated as follows:

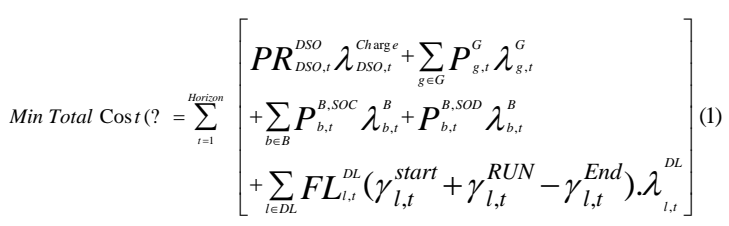

The objective function is represented by an eq. (1), to be minimised is derived from [3], with some modifications to serve our purpose, but the main difference and enhancement is energy cost reduction mechanism employed by the VPP concept to comply with the DSO's shortage requirement in local distribution grids. 
The first term of the objective function $P R_{D S O, t}^{D S O} \lambda_{D S O, t}^{\text {charge }}$ represents the cost related to the quantity of energy the DSO requires at time $t$. The second term $P_{g, t}^{G} \lambda_{g, t}^{G}$ represents the costs associated to power generation at time $t$. The third term $P_{b, t}^{B, S O C} \lambda_{b, t}^{B}$ is the cost of unit $b$ charging at time t. $P_{b, t}^{B, S O D} \lambda_{b, t}^{B}$ denotes the costs of unit $b$ discharging at time $t . F L_{l, t}^{D L} \lambda_{l, t}^{D L}$ denotes the costs of curtailment of adjustable loads at time $t$. The binary variables $\gamma_{l, t}^{\text {START }} / \gamma_{l, t}^{R U N} / \gamma_{l, t}^{E N D}$ are used for the on/off status of disconnectable load curtailment at time $t$.

The following constraints apply to the optimization problem.

DSO request formulation

$\sum_{g \in G} P_{g, t}^{G}+\sum_{l \in F L} F L_{l, t}^{D L}+\sum_{b \in B} P_{b, t}^{S O C}+P_{b, t}^{S O D} \geq P R_{t}^{D S O} \forall t$

Positive flexibility requested by DSO at time $T^{+}$

$\sum_{g \in G} P_{g, t}^{G}+\sum_{l \in F L} F L_{l, t}^{D L}+\sum_{b \in B} P_{b, t}^{S O C}+P_{b, t}^{S O D} \leq-P R_{t}^{D S O} \quad \forall t$

Negative flexibility requested by DSO at time $T$

Battery SOC/D formulation

$P_{b, t}^{\text {injection }} \leq Q_{b}^{\text {injection }} \quad \forall b \in B, t \in T$

Battery state of charge of unit B at time $t$

$P_{b, t}^{\text {withdrawal }} \leq Q_{b}^{\text {withdrawal }} \quad \forall b \in B, t \in T$

Battery state of discharge of unite B at time $t$

Flexible loads formulation

$F L_{l, t}^{D L} \geq 0 \quad \forall l \in F L^{D L}, \forall t$

Scheduled up-regulation

$F L_{l, t}^{D L} \leq 0 \quad \forall l \in F L^{D L}, \forall t$

Scheduled down-regulation

Power limits of distributed generator

$\underline{P_{t}^{G}} v u \leq P_{t}^{G} \leq \overline{P_{t}^{G}} v u \quad \forall g \in P^{G}, \forall t$

$Y_{t}^{G}-Z_{t}^{G}=V_{t}^{G}-V_{(t-1)}^{G} \forall g \in P^{G}, \forall t$

$Y_{t}^{G}+Z_{t}^{G} \leq 1 \forall g \in P^{G}, \forall t$

Network equation

$P_{g, i}^{G}-P R_{d s o, i}^{D S O}=\sum_{j} V_{i, t} V_{j, t}\left[Y_{i, j} \cos \left(\theta_{i, j} \delta_{j, t} \delta_{i, t}\right)\right]$

Bus voltage limits

$V_{i}^{\min } \leq V_{i, t} \leq V_{i}^{\max }$

The graphical visualisation of error analysis of the proposed optimisation problem to find the best possible final result is represented via figure 3 .

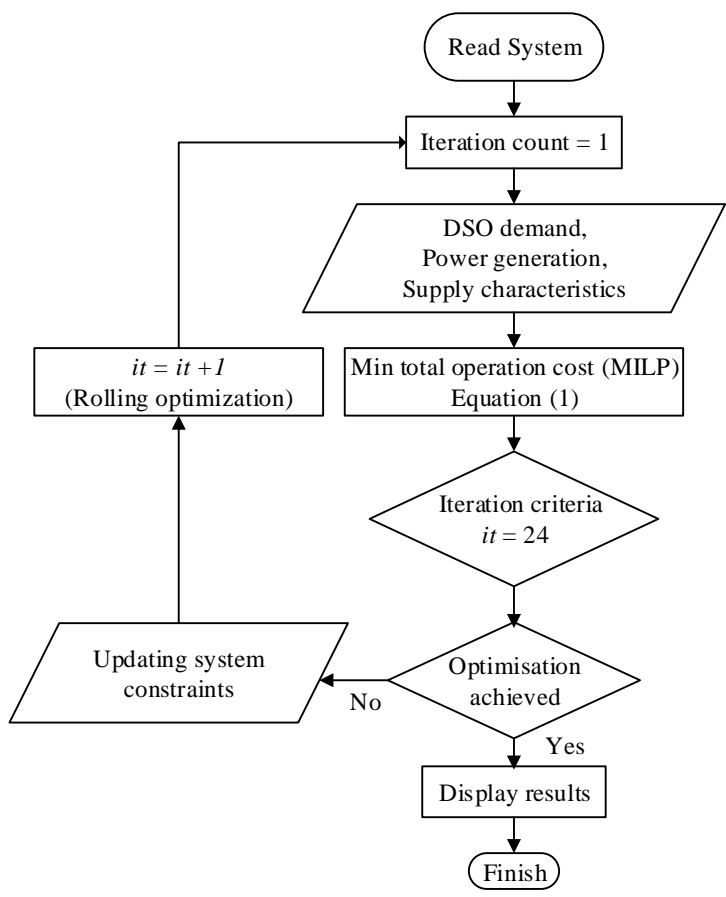

Fig. 3. Optimisation flowchart of the proposed model

\section{Simulation results and discussion}

the test case study presents a small LFM consists of four residences with distributed energy sources. Loads usually include heating systems for room, water heating system, washer-dryer and dish-washer which can be disconnected or shifted under the flexibility contracts. Local generators are solar panels placed on top of the housing roofs. Batteries can be completely remotely operated. In this case the study is based on a weekday resource. The cost of flexibility shall be considered constant for each load and for each supplier throughout the day of operation. The prices of battery charging are taken from DA market. The price data in our case study are taken from the NordPool 2016, Elspot market with some modifications to fit our purpose. The VPP shall apply its daily routine algorithm prior to the start of the day of operation and the DSO shall have submitted its flexibility specifications to the VPP data centre. Results of the proposed approach is validated using the GAMS programming software [14], and the problem is solved by using CPLEX solver on a PC with a core i7 CPU and 16 GB RAM. Optimal active power flow on the local market is being used to minimise operating costs subject to system constraints. The system took $4.023 \mathrm{~s}$ to locate the best possible solution of the test case study.

Fig. 4, clearly demonstrates the overall flexibility shortage requirement requested by the DSO, and it also specifies what flexible assets are scheduled up and or down. The DSO requirement is remunerated through upstream schedule disengaging loads and discharging the batteries. However, on the other hand, the DSO requirement is remunerated during the downstream schedule by reconnecting the loads, charging the batteries and disengaging or decreasing the capacity of PV generators. 


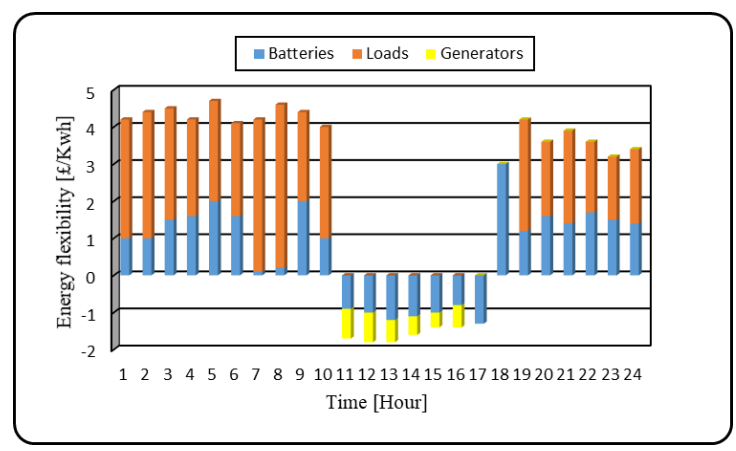

Fig. 4. The DSO energy flexibility request signals

The charging status of a battery is shown via Fig. 5 . $50 \%$ battery status means $2.5 \mathrm{kWh}$, supporting the DSO scheduled up shortage requirement through discharge. Thereafter, charging up to $5 \mathrm{kWh}$ means $100 \%$ charged status supporting downstream regulation retaining energy from photovoltaic cells and releasing it at night. The simulation begins by $50 \%$ of battery SOC and it involves a requirement that the simulation period end with almost the corresponding quantity of energy from the start for the sake of rewarding the impact of the storage systems on the objective function.

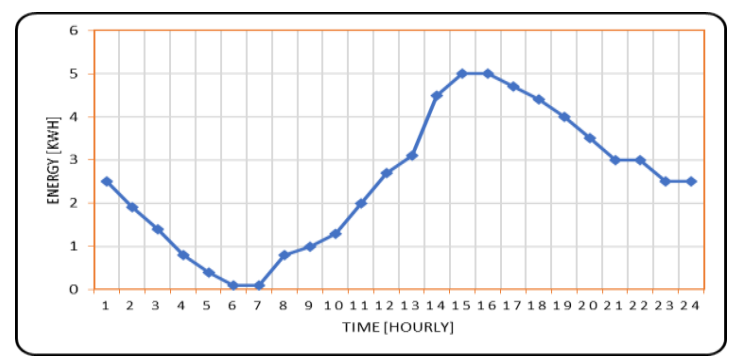

Fig. 5. Battery SOC/discharge

\section{Conclusion and future work}

In this work, a local flexibility market idea is addressed. The LFM approach is taken from previously discussed domains in literature. The aim was to bring together and maintain local energy communities under the umbrella of VPP framework to benefit all. Community participation and involvement in the local energy market can yield more benefits than normal and isolated-mode of energy related services. The initial results of simulation studies have shown that there are network effects occurring.

The implementation of the LFM model would strengthen the active participation of end-users and the share of renewable sources of energy as well. Therefore, the LFM that provides the DSO with local energy flexibility services could be distribution grid upgrade deferral to traditional grid planning decisions.

This work could be further refined by modeling the economic risk of each DER owners impacting the economic welfare of the VPPs. In addition, it would be worth examining the collaboration between load aggregators and VPP. Finally, the inclusion of network constraints into the suggested method will be the subject of future study.

\section{References}

1. D. Thomas, M. Iain, G. Dale, CIRED-Open Access Proceedings Journal, Virtual power plants leveraging energy flexibility in regional markets, $\mathbf{1}$, 2939-2943 (2017)

2. S. Minniti, N. Haque, P. Nguyen, G. Pemen, Energies, Local markets for flexibility trading: Key stages and enablers, 11, 3074 (2018)

3. P. Olivella-Rosell, E. Bullich-Massagué, M. Araguiés-Peñalba, A. Sumper, S.O. Ottesen, A.J. Vidal-Clos, R. Villafáfila-Robles, Applied energy, Optimization problem for meeting distribution system operator requests in local flexibility markets with distributed energy resources, 210, 881-895. (2018)

4. J. Radecke, J. Hefele, L. Hirth, Econstor.eu, Markets for Local Flexibility in Distribution Networks. (2019).

5. S.S. Torbaghan, N. Blaauwbroek, P. Nguyen, M. Gibescu, IEEE $13^{T H}$ International conference on the European Energy Market (EEM), Porto, Portugal, 16 (2016)

6. Y. Li, W. Gao, Y. Ruan, Sustainable cities and society, Feasibility of virtual power plants (VPPs) and its efficiency assessment through benefiting both the supply and demand sides in Chongming country, China, 35, 544-551 (2017)

7. K. Dietrich, L.M. Latorre, L. Olmos, A. Ramos, Electric Power Systems Research, Modelling and assessing the impacts of self-supply and marketrevenue driven Virtual Power Plants, 119, 462-470 (2015)

8. X. Jin, Q. Wu, H. Jia, Applied Energy, Local flexibility markets: Literature review on concepts, models and clearing methods, 261, 114387 (2020)

9. H. Steiniger, In Innovation and Disruption at the Grid's Edge, Virtual Power Plants: Bringing the Flexibility of Decentralized Loads and Generation to Power Markets Academic Press, 331-362 (2017)

10. P. Olivella-Rosell, F. Rullan, P. Lloret-Gallego, E. Prieto-Araujo, R. Ferrer-San-José, S. Barja-Martinez, S.O. Ottesen, IEEE Transactions on Smart Grid, Centralised and Distributed Optimization for Aggregated Flexibility Services Provision, 11, 32573269 (2020)

11. Z. Ullah, G. Mokryani, F. Campean, F.Y. Hu, IET Energy Systems Integration, Comprehensive review of VPPs planning, operation and scheduling considering the uncertainties related to renewable energy sources. 1, 147-157 (2019)

12. W.S. Sakr, H.A. Abdel-Ghany, R.A. EL-Sehiemy, A.M. Azmy, Alexandria Engineering Journal, Techno-economic assessment of consumers' participation in the demand response program for optimal day-ahead scheduling of virtual power plants. 59, 399-415 (2020)

13. L.F. van Summeren, A.J. Wieczorek, G.J. Bombaerts, G.P. Verbong, Energy Research \& 
Social Science, Community energy meets smart grids: Reviewing goals, structure, and roles in Virtual Power Plants in Ireland, Belgium and the Netherlands, 63, 101415 (2020)

14. D. Chattopadhyay, IEEE Transactions on Power Systems, Application of general algebraic modeling system to power system optimization, 15-22. (1999). 